\title{
Polarization properties of photonic bandgap fibers
}

\section{Broeng, Jes; Libori, Stig E. Barkou; Bjarklev, Anders Overgaard}

\section{Published in:}

Optical Fiber Communication Conference 2000

Link to article, DOI:

10.1109/OFC.2000.868535

Publication date:

2000

Document Version

Publisher's PDF, also known as Version of record

Link back to DTU Orbit

Citation (APA):

Broeng, J., Libori, S. E. B., \& Bjarklev, A. O. (2000). Polarization properties of photonic bandgap fibers. In Optical Fiber Communication Conference 2000 (Vol. 3, pp. 101-103). https://doi.org/10.1109/OFC.2000.868535

\section{General rights}

Copyright and moral rights for the publications made accessible in the public portal are retained by the authors and/or other copyright owners and it is a condition of accessing publications that users recognise and abide by the legal requirements associated with these rights.

- Users may download and print one copy of any publication from the public portal for the purpose of private study or research.

- You may not further distribute the material or use it for any profit-making activity or commercial gain

- You may freely distribute the URL identifying the publication in the public portal 
ThG2-1/101

\title{
Polarization properties of photonic bandgap fibers
}

\author{
Jes Broeng, Stig E. Barkou, Anders Bjarklev \\ Center for Communications, Optics, and Materials (COM) \\ Technical University of Denmark, Building 349, DK-2800 Lyngby, Denmark \\ Telephone: +4545881444,Fax:+4545936581, E-mail: jb@com.dtu.dk
}

Transmission of light in optical fibers are traditionally based on total internal reflection (TIR). A high-index core surrounded by a low-index cladding has been the most basic requirement for all long-distance optical waveguides. However, within the last two years a novel class of optical fibers, allowing leakage-free guidance of light in a low-index core region, has emerged [1-4]. These fibers are operating by photonic bandgap (PBG) effects, which occur due to a periodic microstructuring of air holes in the cladding region [5]. Such PBG-fibers possess a number of properties separating them from conventional TIR-based fibers $[1,4,6]$ - and represent a great potential for special fibers. To investigate the potential of PBG-fibers as basic transmission medium in future long-distance optical communication systems, we have previously presented an analysis of their dispersion properties [7], and found that they may be designed to exhibit flat, near-zero dispersion over a broad wavelength range, or to exhibit a high anomalous dispersion while remaining single moded. To further investigate the potential of PBG-fibers, we present in this work an analysis of their polarization properties.

For high-speed, optical communication systems based on conventional fibers even very low degrees of birefringence $\left(<10^{-7}\right)$ may cause a crucial dispersion differences between the two polarization modes. Therefore, it is vital to predict and understand the polarization effects which may result from non-uniformities in and around the core region of a PBG-fiber. At present, no experimental studies of birefringence in PBG-fibers have been presented in the literature, and all theoretical investigations have been based on PBG-fibers with ideal structures - having no unintentional imperfections or asymmetries. To analyze the polarization effects resulting from structural non-uniformities, a honeycomb-based PBG-fiber design was chosen as this was the first in which PBG-waveguidance was demonstrated. The design is illustrated in Fig. 1, where non-uniformities were simulated by breaking the 60 degree symmetry of the structure in the immediate proximity to the center-hole defect. For the specific fibers analyzed here, a $10 \%$ diameter difference was introduced for the two air holes $P$ compared to the remainder of holes in the fiber structure.

A modal index analysis of three different fibers is presented in Fig. 2(a). For all three fibers the holes in the periodic cladding structure have a diameter, $d_{c l}$, of $0.40 \Lambda$ and a similar sized center hole. For the 'ideal' fiber, the holes $P$ are identical to the cladding holes, while the type 1 fiber has $P$ holes with a diameter $d_{P}=0.44 \Lambda$, and the type 2 fiber has $d_{P}=0.36 \Lambda$. The numerical method used for the simulation was a plane-wave, variational method [6,8], employing 16384 plane waves to represent the field solutions. For the fiber type 1 (with increased hole sizes), the figure indicates a slight shift in the mode index towards the lower bandgap edge, i.e. towards lower index values. This may readily be understood from the fact that the imperfection causes a small increase in the air filling fraction of the core region. In accordance with this, the mode index for the fiber type 2 is shifted towards higher index values. As is apparent, these shifts do not cause significant alterations in the operational window of the fibers, which remains approximately from $\Lambda / \lambda=0.7$ to 1.4 , where $\Lambda$ is the center-to-center distance between two holes in the periodic cladding structure and $\lambda$ is the free-space wavelength. However, the non-uniformities do result in important changes in the polarization properties of the fibers. As for conventional TIR-fibers, the guided mode for each of 


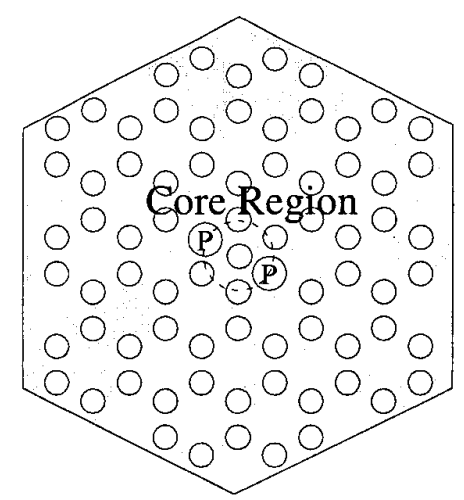

Fig. 1. Schematic illustration of the fiber cross-section of the basis structure used for the analysis of polarization properties of honeycomb PBG-fibers having non-uniformities in the core region.

the fibers in Fig.2(a) consists of two polarization modes. While these two modes for the ideal fiber - as expected - are degenerate, the degeneracy of the guided mode is strongly lifted for both fiber type 1 and fiber type 2. This birefringence, introduced solely from the $10 \%$ diameter-change of the holes, $P$, is for fiber type 1 illustrated in Fig.2(b). The figure reveals a birefringence on the

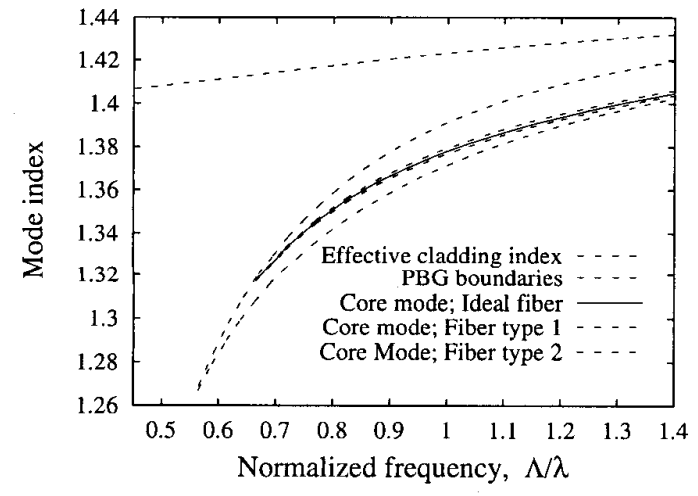

(a)

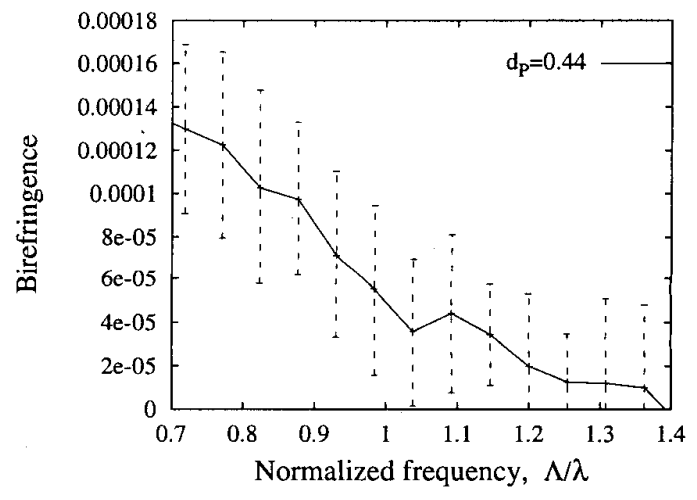

(b)

Fig. 2. (a) Modal index analysis of three types of fibers. The ideal fiber has identical air holes with a size $d_{c o}=d_{c l}=d_{P}=0.4 \Lambda$, Fiber type 1 has $d_{c o}=d_{c l}=0.40 \Lambda, d_{P}=0.44 \Lambda$, and Fiber type 2 has $d_{c o}=d_{c l}=0.40 \Lambda$, $d_{P}=0.36 \Lambda$. (b) Birefringence for fiber type 1 .

order of $10^{-5}$ over the operational window of the fiber. The fiber type 2 showed a slightly less degree of birefringence. Since non-uniformities are almost unavoidable with the present technology for fabricating PBG-fibers, the relatively large birefringence occurring from the modest diameter changes for the two holes $P$ indicates a potential problem for the utilization of PBG-fibers as the basic transmission medium in a long-distance, high bit-rate, optical communication link. On the other hand, the high degree of birefringence points strongly towards applications of PBG-fibers as polarization maintaining devices.

Based on the above discussion, it may at present seem most relevant to explore the high- 
birefringence potential of PBG-fibers. With this aim, a final fiber was analyzed having $d_{c o}=d_{c l}=0.40 \Lambda$, $d_{P}=0.44 \Lambda$, and the positions of the holes $P$ shifted a distance $d_{P} / 2$ towards the center-defect. As the properties of the guided mode is mainly determined by the core geometry - while the cladding structure determines the wavelength range in which the fiber can be operated - a change in position towards the center was expected to have the largest effect on the polarization properties. The birefringence for this fiber was found to be as large as $5 \cdot 10^{-4}$ at a normalized frequency, $\Lambda / \lambda$, of 0.9. The field distributions of the two orthogonal polarization modes are illustrated in Fig.3.
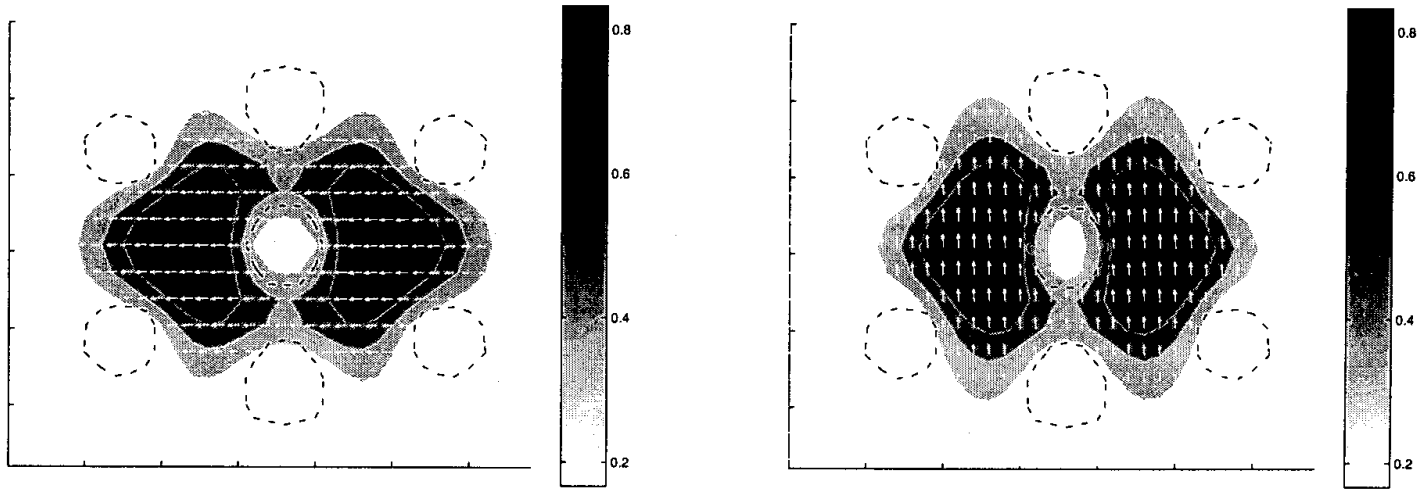

Fig. 3. Illustration of the field distribution of the two orthogonal polarisation modes (dark areas indicate high intensity). The fibre geometry is indicates by the dashed lines, and the arrows illustrate the transverse vector components.

In conclusion, we have presented the first investigations of polarization properties of optical fibers operating by PBG effects. Strong birefringence were seen to result from moderate nonuniformities in and around the core region. For one particular fiber, a simple, first optimization was performed, and the fiber was found to display a high birefringence of $5 \cdot 10^{-4}$. This early study of polarization properties of PBG-fibers, therefore, suggests utilization of PBG-fibers for polarization maintaining and manipulating purposes - but also indicate a potential limitation to their use as a basic transmission medium for long-distance optical communication.

\section{REFERENCES}

[1] J. Broeng, D. Mogilevtsev, S.E. Barkou, and A. Bjarklev. Photonic crystal fibres: a new class of optical waveguides. Optical Fiber Technology, 5:305-30, July 1999. (invited paper) In press.

[2] S.E. Barkou, J. Broeng, and A. Bjarklev. Silica/air photonic crystal fiber design that permits waveguiding by a true photonic bandgap effect. Optics Letters, 24(1):46-8, Jan. 1999.

[3] J.C. Knight, J. Broeng, T.A. Birks, and P.St.J. Russell. Photonic band gap guidance in optical fibers. Science, 282(5393):1476-1478, Nov. 201998.

[4] R.F. Cregan, B.J. Mangan, J.C. Knight, T.A. Birks, P.St.J. Russell, P.J. Roberts, and D.C. Allan. Single-mode photonic band gap guidance of light in air. Science, 285:1537-9, Sept. 1999.

[5] J. Broeng, S.E. Barkou, A. Bjarklev, J.C. Knight, T.A. Birks, and P.St.J. Russell. Highly increased photonic band gaps in silica/air structures. Optics Communications, 156(4-6):240-4, Nov. 1998.

[6] J. Broeng, T. Søndergaard, S.E. Barkou, P.M. Barbeito, and A. Bjarklev. Waveguidance by the photonic bandgap effect in optical fibres. Pure and Applied Optics, 1:477-82, July 1999.

[7] S.E. Barkou, J. Broeng, and A. Bjarklev. Dispersion properties of photonic bandgap guiding fibers. In Optical Fiber Communication Conference, San Diego, pages 117-9, Feb. 1999. FG5.

[8] R.D. Meade, A.M. Rappe, K.D. Brommer, J.D. Joannopoulos, and O.L. Alerhand. Accurate theoretical analysis of photonic band-gap materials. Physical Review B, 48(11):8434-7, Sept. 1993. 